European Federation of Societies for Ultrasound in Medicine and Biology

\title{
EUROSON 2017 EFSUMB Young Investigator Prize Winners
}

First Prize - Clinical Presentation

Matteo Garcovich - Italy

Joint First Prize - Technical Presentation

Ruud JG van Sloun - The Netherlands

Maria Theodorou - UK

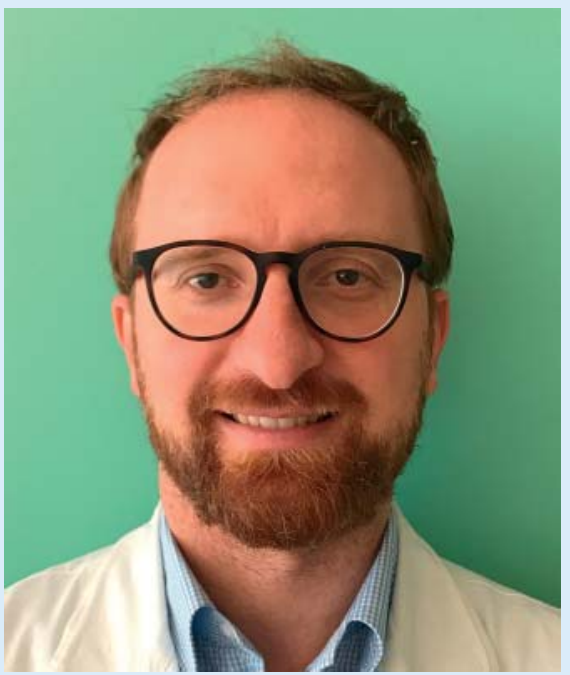

Matteo Garcovich

Matteo Garcovich received his medical degree at the "A. Gemelli" Hospital in Rome. He later became an Internal Medicine Specialist and obtained a PhD at the same University, having spent a year as a clinical research fellow at the Royal Free Hospital (London, UK) during his speciality training. In the last years he (co-) authored 27 publications in ISI journals and has won several Young Investigator awards at the annual conferences of EASL, UEG and SIUMB. His main fields of interest include liver elastography, contrast-enhanced ultrasound and portal hypertension. He speaks Italian, German and English and has, collaterally, an academic degree in Violin.

\section{Abstract}

\section{Assessing Baveno VI criteria with a new point-shear wave elastography technique: the BAVElastPQ study}

Purpose

To date no study has explored the potential role of ElastPQ, a novel point-SWE technique, in the assessment of clinically signifiant portal hypertension. The aim of our study was to determine a liver stiffness (LS) cut-off value measured by ElastPQ and/or laboratory parameters that could help identify those patients who can safely avoid screening endoscopy, similarly to the recently proposed Baveno VI criteria which recommends a $L S$ value $<20 \mathrm{kPa}$ measured by transient elastography in combination to a platelet count $>150,000 / \mu \mathrm{l}$.

\section{Methods}

Data was collected on 1385 patients who underwent ElastPQ measurement from January 2013 to January 2016 in our Department. Inclusion criteria were a LS value of $\geq 7 \mathrm{kPa}$ (to reasonably rule-in all patients with advanced fibrosis and cirrhosis) and an upper gastrointestinal endoscopy within 12 months, with a diagnosis of compensated chronic liver disease. Exclusion criteria were history of decompensated liver disease, evidence of porto-spleno-mesenteric vein thrombosis and non-cirrhotic portal hypertension. Varices were graded as low risk (grade $<2$ ) or high risk (grade 22 ).

\section{Results}

The study included 184 patients (114[62\%] HCV, and 160[87\%] Child-Pugh A). Varices were present in $36 \%$ cases, with $10 \%$ prevalence of high-risk varices. According to ROC curve analysis LS measurement and platelet count were evaluated as predictors of high-risk varices. Overall 74/184 (40\%) met the "BAVElastPQ" criteria (that is, LS $<12 \mathrm{kPa}$ and platelet count $>150,000 / \mu \mathrm{l}$ ). Within this group $11 / 63(17 \%)$ had any grade of varices and only $1 / 73(1 \%)$ had high-risk varices. The BAVElastPQ criteria gave sensitivity of 0.95 , specificity of 0.44 , positive predictive value of 0.16 and negative predictive value of 0.98 . The AUROC for LS and platelet count was 0.81 and 0.76 , respectively.

\section{Conclusion}

The BAVElastPQ criteria correctly identified $99 \%$ of patients without high-risk varices. By applying such criteria we could have potentially avoided $40 \%$ surveillance endoscopies in our cohort.

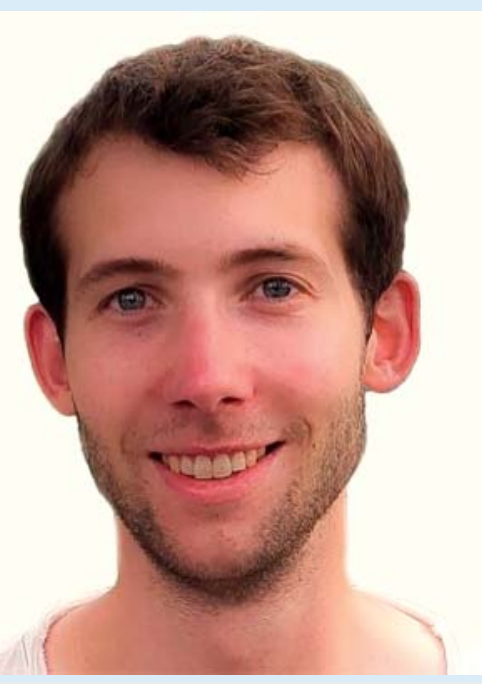

Ruud J. G. van Sloun

Ruud J. G. van Sloun was born in Roermond, The Netherlands, in 1990 . He received the B.Sc. and M.Sc. degree (with distinction cum laude) in electrical engineering from the Eindhoven University of Technology, The Netherlands, in 2012 and 2014, respectively, where he is currently pursuing the Ph. D. degree. In 2013-2014, he was 
an intern at Philips Research Eindhoven, specializing in the field of medical ultrasound imaging and nonlinear acoustics. He has authored over 30 publications including 12 papers in international peerreviewed scientific journals. His current research interests include quantitative (contrast enhanced) ultrasound imaging in oncology, machine learning, compressed sensing, signal and image processing, and sensor analytics.

\section{Abstract}

\section{Prostate cancer localization with contrast-enhanced ultrasound - from technical innovation to clinical implementation}

\section{Purpose}

Prostate cancer (PCa) is the second-leading cause of cancer death in men, and the most frequently diagnosed cancer in men aside from skin cancer [1]. PCa diagnosis is typically performed using systematic needle biopsies. Despite being the current golden standard, initial systematic biopsies miss nearly a quarter of the clinically significant cancers, requiring additional biopsy sessions in case of suspicion. To enhance cancer detection rates, targeting of biopsies using multi-parametric magnetic resonance imaging (mpMRI) has been proposed. Accurate imaging of lesions would also enable the use of minimally invasive focal therapies as opposed to radical intervention. Nevertheless, mpMRI is a modality associated with high costs and a complex workflow.

Angiogenesis is known to play a central role in the growth of tumours towards a metastatic or a lethal phenotype. It is characterized by the formation of a chaotic network of microvessels feeding the tumour. With the aim of detecting those changes in the vascular architecture that are associated with cancer, we have introduced a wide spectrum of advanced contrast-enhanced ultrasound (CEUS) technologies for quantification of the chaotic nature of tumour vasculature.

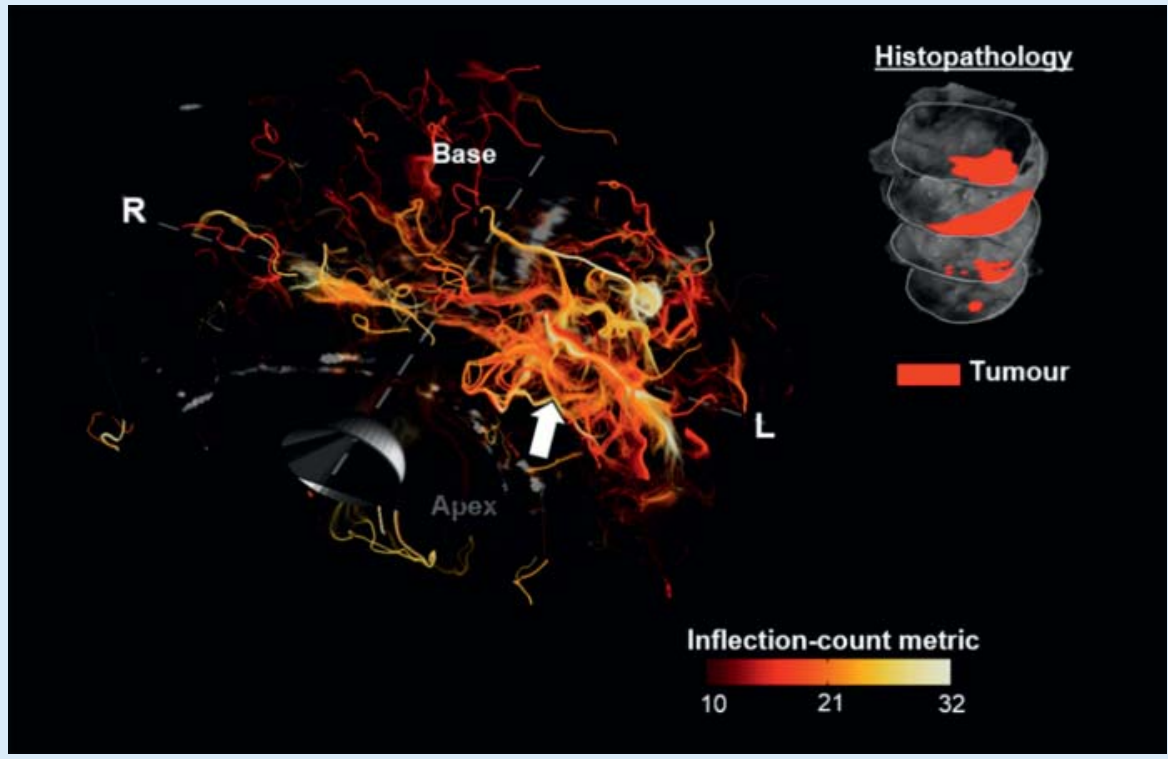

- Fig. 13 D Contrast-enhanced ultrasound tractography (CEUS-T) and tortuosity quantification on a human prostate with corresponding histology. Colours encode the tract tortuosity by the inflection count metric. CEUS-T displays elevated tortuosity and higher tract-density on the left mid-base side of the prostate. Similarly, histology reveals a significant malignant lesion (Gleason grade: $4+5)$, with a left mid-basal focus.

\section{Methods}

In [2], we proposed for the first time the characterization of the microvascular network by local system identification, enabling the estimation of flow velocity and contrast dispersion. Both parameters were shown to be correlated to the presence of malignant tissue. Later [3], this work was extended by estimating the full vascular flow vector field across the organ. The heterogeneity of these flow patterns was higher in cancer. When testing the method on 24 patients referred for radical prostatectomy, its quantification through the local vector field entropy yielded a cancer classification accuracy of $82 \%$, outperforming the traditional perfusion parameters by almost $10 \%$.

More recently, we developed CEUS-tractography, which combines revolutionary concepts from diffusion tensor tractography MRI for fibre visualization with CEUS blood flow vector imaging to yield $3 \mathrm{D}$ images of contrast agent trajectories using a clinical ultrasound scanner. An example of CEUStractography applied to clinically acquired 3 D CEUS data is provided in $>$ Fig. 1.

\section{Results}

All these methods have shown their promise in a preliminary validation. To extend beyond technical innovation and make the translation towards clinical impact, we are currently investigating the clinical value of these techniques for CEUS-targeted biopsies in a clinical study [4] at the Academic Medical Centre (University of Amsterdam, Amsterdam, The Netherlands). Although this study is still on-going, initial results on 49 patients suggest advanced CEUS alone to produce tumour detection rates similar to complex, costly, and time-consuming mpMRI-targeted biopsies.

\section{References}

[1] American Cancer Society, "Cancer Facts \& Figures 2015”, American Cancer Society, Atlanta, 2015

[2] van Sloun RJG, et al., Medical Image Analysis, 2017; 35: 610-619

[3] van Sloun RJG, et al., IEEE transactions on medical imaging, 2017; 36(3): 826 - 837

[4] Postema AW, et al., BMC Urology, 2017 


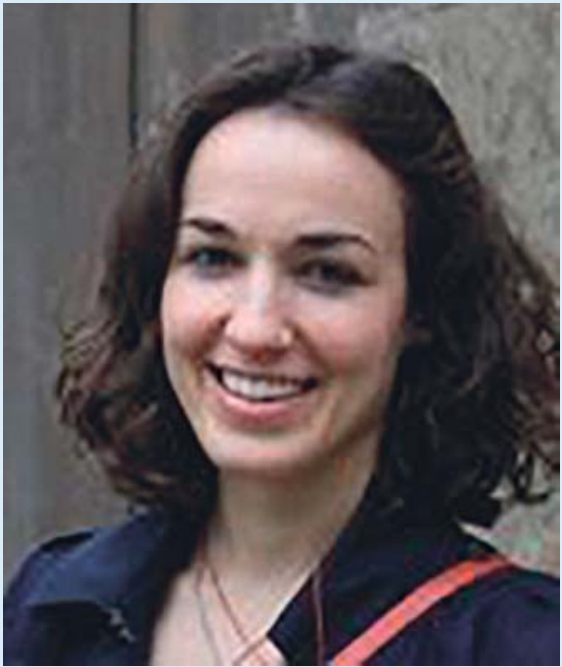

Maria Theodorou

Maria Theodorou received her MSc in Biomedical Engineering from Imperial College London in 2010 and after working in the private sector the following two years, she joined the four year doctoral programme of the Institute of Cancer Research, University of London, where she completed her PhD in Medical Physics funded by Cancer Research UK. She is currently a postdoctoral researcher at the Institute of Cancer Research and Royal Marsden Hospital, working in the area of quantitative ultrasound imaging and elastography methods.

\section{Abstract}

\section{Permelastography: Quantitative poroelastic property imaging by combining shear wave and strain Elastography}

Purpose

Poroelastic theory may be used to explain both cellular and tissue rheology. It describes the movement of fluid away from a region of tissue during sustained compression. The resulting time- and spatially varying deformation of the tissue matrix may be imaged as a change in ultrasound echo strain over time. Strain movies allow the quantitative imaging of the product of the tissue's aggregate modulus and fluid permeability, Hk, and its Poisson's ratio, v. We have successfully developed a novel ultrasound technique to measure tissue fluid permeability, $\mathrm{k}$, independently of $\mathrm{H}$, which we have assessed on tissue-mimicking phantoms.

\section{Methods}

In the new method, which we call permelastography, $\mathrm{H}$ is calculated from $\mathrm{v}$ and the tissue's Young's modulus, $\mathrm{E}$, where $\mathrm{E}$ is obtained from shear wave elastography (SWE). This was tested on a series of agarose phantoms of varying matrix pore size, controlled by varying agarose concentration. By combining the Hk, $\mathrm{v}$ and $\mathrm{E}$ maps, the permeability was extracted and varied systematically and reproducibly with matrix pore size. Intra-phantom variation in $\mathrm{k}$ was about $15 \%$ of the mean, and the mean values of $\mathrm{k}$ fell within about $28 \%$ of those measured using an independent but destructive method.

\section{Results \& Conclusion}

The non-invasive estimation of the biomechanical properties $\mathrm{E}, \mathrm{k}$ and $\mathrm{v}$ could be valuable clinically because cancer is associated with altered ability of fluid to move in tissue due to angiogenesis. The experimental setup was adapted into a light, $3 \mathrm{D}$ printed handheld design comprising one ultrasound and one SWE transducer jointly held above a compressor plate, which provides force-feedback to the operator. The prototype has been evaluated clinically to characterize the changes in the poroelastic behavior of the breast of healthy volunteers due to the hormonal response of tissue fluid flow in different phases of the menstrual cycle.

Key words: permeability, elastography, breast 